\title{
DISPLASIA DA VALVA TRICÚSPIDE EM UM CÃO DA RAÇA SHIH-TZU
} Nathana Beatriz Martins ${ }^{1}$, Gustavo Cavinato Herrera ${ }^{2}$, Christina de Siqueira Mendonça $^{2}$,
Vanessa Martins Fayad Milken ${ }^{4}$.

${ }^{1}$ Médica Veterinária Residente do Hospital Veterinário da Universidade Federal de Uberlândia (UFU), Uberlândia-MG, Brasil. E-mail: nathanabmartins@gmail.com

${ }^{2}$ Médico Veterinário do Hospital Veterinário da UFU, Uberlândia-MG, Brasil.

${ }^{3}$ Médica Veterinária do Hospital Veterinário da UFU, Uberlândia-MG, Brasil.

${ }^{4}$ Professora Doutora de Diagnóstico por Imagem da UFU, Uberlândia, Brasil.

Recebido em: 06/04/2019 - Aprovado em: 10/06/2019 - Publicado em: 30/06/2019 DOI: 10.18677/EnciBio_2019A57

A displasia da valva tricúspide é uma cardiopatia congênita, pouco frequente em cães e não relatado em cães da raça shih-tzu. É caracterizada pelo espessamento focal ou difuso dos folhetos valvulares, subdesenvolvimento das cordoalhas tendíneas e músculos papilares, separação incompleta dos componentes valvares da parede ventricular, além da agenesia do tecido valvular. O objetivo deste relato foi descrever os sinais clínicos, as alterações ultrassonográficas e ecocardiográficas de um cão da raça shih-tzu de três meses, macho, apresentando prostração, anorexia, dispneia, vômito, diarreia e aumento de volume abdominal. Amostras de sangue foram coletadas e houve anemia macrocítica e normocrômica, desvio a esquerda regenerativo e linfopenia, além de hipoproteinemia por hipoalbuminemia. O valor de creatinina estava dentro dos parâmetros normais. $O$ exame coproparasitológico das fezes apresentou-se negativo pelo método direto e Willis. Ao exame ultrassonográfico, o baço, fígado e ambos os rins apresentaram-se alterados, com presença de liquido anecóico sem celularidade, livre no abdômen em quantidade moderada, sugestivo de transudato ou ascite. No exame ecocardiográfico, foi possível observar dilatação atrial e ventricular direita, com regurgitação de tricúspide, valva tricúspide de aspecto anormal, com folheto septal preso ao septo e folheto anterior maior que o normal e movimentação paradoxal do septo devido à sobrecarga de volume do ventrículo direito. Todas as alterações encontradas no presente relato corroboram com as descritas em literatura.

PALAVRAS-CHAVE: cardiopatia, congênito, coração.

\section{TRICUSPID VALVE DYSPLASIA IN A DOG SHIH-TZU}

\begin{abstract}
Tricuspid valve dysplasia is a congenital heart disease, uncommon in dogs and not reported in shih-tzu dogs. It is characterized by focal or diffuse thickening of valvular leaflets, underdevelopment of tendon chordae and papillary muscles, incomplete separation of valvular components from the ventricular wall, and agenesis of valvular tissue. The objective of this report was to describe the clinical signs, sonographic and echocardiographic changes of a 3-month-old male shih-tzu dog presenting with prostration, anorexia, dyspnea, vomiting, diarrhea and abdominal volume increase. Samples of blood were collected and there was macrocytic and
\end{abstract}


normochromic anemia, regenerative left deviation and lymphopenia, as well as hypoalbuminemia hypoproteinemia. The creatinine value was within the normal range. The coproparasitological exam of the faeces was negative by the direct method and Willis. At sonographic examination, the spleen, liver and both kidneys were altered, with the presence of anechoic fluid without cellularity, free in the abdomen in a moderate amount, suggestive of transudate or ascites. At the echocardiographic examination, it was possible to observe atrial and right ventricular dilatation, with tricuspid regurgitation, abnormal tricuspid valve with septal leaflet attached to the septum and anterior leaflet larger than normal, and paradoxical movement of the septum due to right ventricular volume overload. All alterations found in the present report corroborate with those described in the literature.

KEYWORDS: heart disease, congenital, dog.

\section{INTRODUÇÃO}

A displasia da válvula tricúspide consiste em uma anomalia no aparato valvar atrioventricular direito. É uma cardiopatia congênita pouco frequente em cães (NELSON; COUTO, 2009; ETTINGER; FELDMAN, 2010; OLIVEIRA et al., 2011; BEIJERINK et al., 2017). Na medicina humana, está associada à alta mortalidade em crianças (WANG et al., 2018). Em um estudo retrospectivo realizado na Itália, por Oliveira et al. (2011), de 4480 cães com doenças cardíacas, 976 animais tinham cardiopatia congênita $(21,7 \%)$. Destes, apenas 35 apresentaram displasia da válvula tricúspide (3,1\%). Porém, no Brasil, a frequência desta afecção não é conhecida, podendo haver variação na incidência e nas raças mais acometidas de acordo com cada região (SOUSA et al., 2006).

A maioria dos cães afetados são jovens, de raças puras, de grande porte, com destaque para as raças Labrador, Pastor Alemão e Boxer, sendo os machos mais acometidos (OLIVEIRA et al., 2011). Segundo Liu; Tilley (1976), a displasia da valva tricúspide já foi relatada em cães da raça Pastor Alemão, Dogue Alemão, Setter Irlandês, Boxer, Old English Sheepdog e Weimaraner, embora o Labrador Retriever, seja a raça mais acometida (BONAGURA, 1989; MOISE, 1989). Nessa raça, relatou-se a transmissão genética (MEURS, 2010).

As lesões descritas, em seres humanos, incluem espessamento focal ou difuso dos folhetos valvares, subdesenvolvimento das cordas tendíneas e músculos papilares, separação incompleta dos componentes valvares da parede ventricular e agenesia focal do tecido valvular (BECKER et al., 1971). Em cães e gatos, os músculos papilares geralmente estão aumentados e as cordas tendíneas curtas ou ausentes (LIU; TILLEY 1976).

Como consequência, os músculos papilares aumentados com frequência se fixam diretamente aos folhetos valvares, travando-os ao ventrículo e impedindo o fechamento sistólico. Com frequência, os folhetos estão irregularmente espessados e podem conter fenestrações. O folheto septal pode aderir parcialmente ao septo ventricular (OLIVIER, 1988). A displasia de tricúspide grave causa dilatação acentuada do átrio direito, pressão atrial direita aumentada e pode levar a insuficiência cardíaca direita com ascite e efusão pleural. Entretanto, uma regurgitação tricúspide não complicada, leve a moderada, pode ser bem tolerada durante muitos anos e permitir qualidade de vida (NYLAND; MATTOON, 2005; BEIJERINK et al., 2017).

O sinal clínico mais comum é a presença de um sopro holossistólico na auscultação do foco tricúspide (FOX et al., 1999; NELSON; 
COUTO, 2009). O ecocardiograma mostra alterações na forma, tamanho e movimentação dos folhetos da válvula, podendo também avaliar a regurgitação com o auxílio do Doppler (BOON, 2005).

Geralmente, o exame em modo-M revela anormalidades não especificas, mas muito sugestivas, incluindo a dilatação acentuada do lado direito do coração sem espessamento da parede e a aparência de uma câmara ventricular comparativamente pequena. O movimento paradoxal do septo também é um achado comum. Pelo fato de a acentuada dilatação atrial direita causar deslocamento ventral do ventrículo direito, o feixe do modo-M frequentemente passa através do átrio direito, em vez do ventrículo direito, resultando na aparência de uma larga e fina parede proximal da câmara. Anormalidade valvares geralmente são indistinguíveis apenas pelo exame em modo-M (NYLAND; MATTOON, 2005).

O exame bidimensional permite o reconhecimento, de modo mais claro, do átrio e do ventrículo direitos dilatados e do achatamento diastólico septal, e um exame mais completo da válvula tricúspide (NYLAND; MATTOON, 2005). As imagens ecocardiográficas que permitem melhor avaliação da valva tricúspide são as obtidas no corte paraesternal longitudinal direito e apical quatro câmaras no corte paraesternal longitudinal esquerdo (MADRON et al., 2015). Com relação aos componentes valvares, pode-se observar a valva septal unida ao lado direito do septo interventricular por cordas tendíneas curtas. É frequente visualizar a valva parietal da tricúspide alongada, com uma união anormal a uma rede de músculos papilares, assim como a não união dos folhetos valvares (BOON, 2011).

De acordo com Sousa et al. (2006), a displasia de tricúspide pode estar associada à Anomalia de Ebstein, que é caracterizada pela inserção mais baixa do aparelho valvar na parede ventricular direita, próximo ao ápice. Esta condição causa aumento exacerbado de tamanho do átrio direito, tornando o ventrículo direito diminuído, condição denominada de ventrículo atrializado (BOON, 2011).

As radiografias torácicas mostram aumento de câmaras cardíacas direitas, com coração em "D" invertido na projeção ventrodorsal (THRALL, 2010). Onda P pulmonale, sinais de sobrecarga do ventrículo direito e desvio do eixo cardíaco médio para a direita podem estar presentes no eletrocardiograma (FILIPPI, 2011).

O prognóstico é de reservado a ruim e o comprometimento hemodinâmico progressivo é comum (TIERNEY et al., 2017). Na medicina humana, os casos menos graves, a doença corresponde a um risco médio de mortalidade de apenas 1 a $1,4 \%$ ao ano em crianças (MALVINDI; VIOLA, 2015). Nos cães, a prevalência inicial é desconhecida. Em cães jovens e adultos, o prognóstico é semelhante ao dos humanos e depende principalmente da gravidade da malformação. Cães com pequenas insuficiências geralmente têm uma vida normal (BEIJERINK et al., 2017). O objetivo do presente estudo é relatar o caso de um cão da raça shih-tzu com displasia de valva tricúspide.

\section{RELATO DE CASO}

Um cão, da raça shih-tzu, três meses de idade, macho, pesando $1,100 \mathrm{~kg}$, foi resgatado em ambiente urbano e encaminhado ao Hospital Veterinário da Universidade Federal de Uberlândia (HV-UFU). O animal apresentava prostração, anorexia, dispneia, vômito e diarreia, aumento de volume abdominal. No exame físico, observou-se desidratação, mucosas hipocoradas, escore de condição corporal $5 / 9$, temperatura $35,4^{\circ} \mathrm{C}$, frequência cardíaca 140 batimentos por minuto (bpm) e frequência respiratória 36 movimentos por minuto ( $\mathrm{mpm})$. 
$\mathrm{Na}$ auscultação cardíaca, foi detectado sopro em tricúspide. O abdômen estava abaulado, com presença de líquido.

Amostras de sangue foram coletadas, caracterizando anemia macrocítica e normocrômica, desvio a esquerda regenerativo e linfopenia, além de hipoproteinemia por hipoalbuminemia. O valor de creatinina estava dentro dos parâmetros normais. O exame coproparasitológico das fezes apresentou-se negativo pelo método direto e Willis. $\mathrm{O}$ animal ficou internado e como tratamento, optou-se por ondansetrona $0,5 \mathrm{mg} / \mathrm{kg}$, metronidazol $15 \mathrm{mg} / \mathrm{kg}$, ranitidina $2 \mathrm{mg} / \mathrm{kg}$, furosemida $4 \mathrm{mg} / \mathrm{kg}$, enalapril $1 \mathrm{mg} / \mathrm{kg}$ e pimobendan $0,3 \mathrm{mg} / \mathrm{kg}$.

No exame ultrassonográfico, a bexiga apresentou-se tópica, com repleção média, contorno definido, conteúdo anecoico, parede interna regular e delgada. $\mathrm{O}$ baço com formato anatômico preservado, dimensões discretamente aumentadas, parênquima homogêneo e hiperecoico, sugestivo de congestão e inflamação. Ambos os rins, apresentaram formato anatômico e dimensões preservadas, arquitetura interna alterada, relação corticomedular irregular, cortical delgada, hipoecoica com ecotextura preservada, sinal da margem medular, divertículos irregulares e região medular heterogênea, sugestivo de nefrite congênita.

O fígado apresentava-se aumentado, com bordos arredondados, parênquima homogêneo e bastante hiperecoico, ecotextura preservada, vias biliares sem evidencias de alterações e vasos moderadamente dilatados, sugestivo de congestão, colestase e hepatite. A vesícula biliar vazia, de contornos definidos, pouco conteúdo anecoico, parede interna regular, delgada e hiperecoica. As alças intestinais de estratificação preservada e presença de gases. A próstata não foi visualizada. Presença de líquido anecóico sem celularidade, livre no abdômen em quantidade moderada, sugestivo de transudato ou ascite (Figura 1).

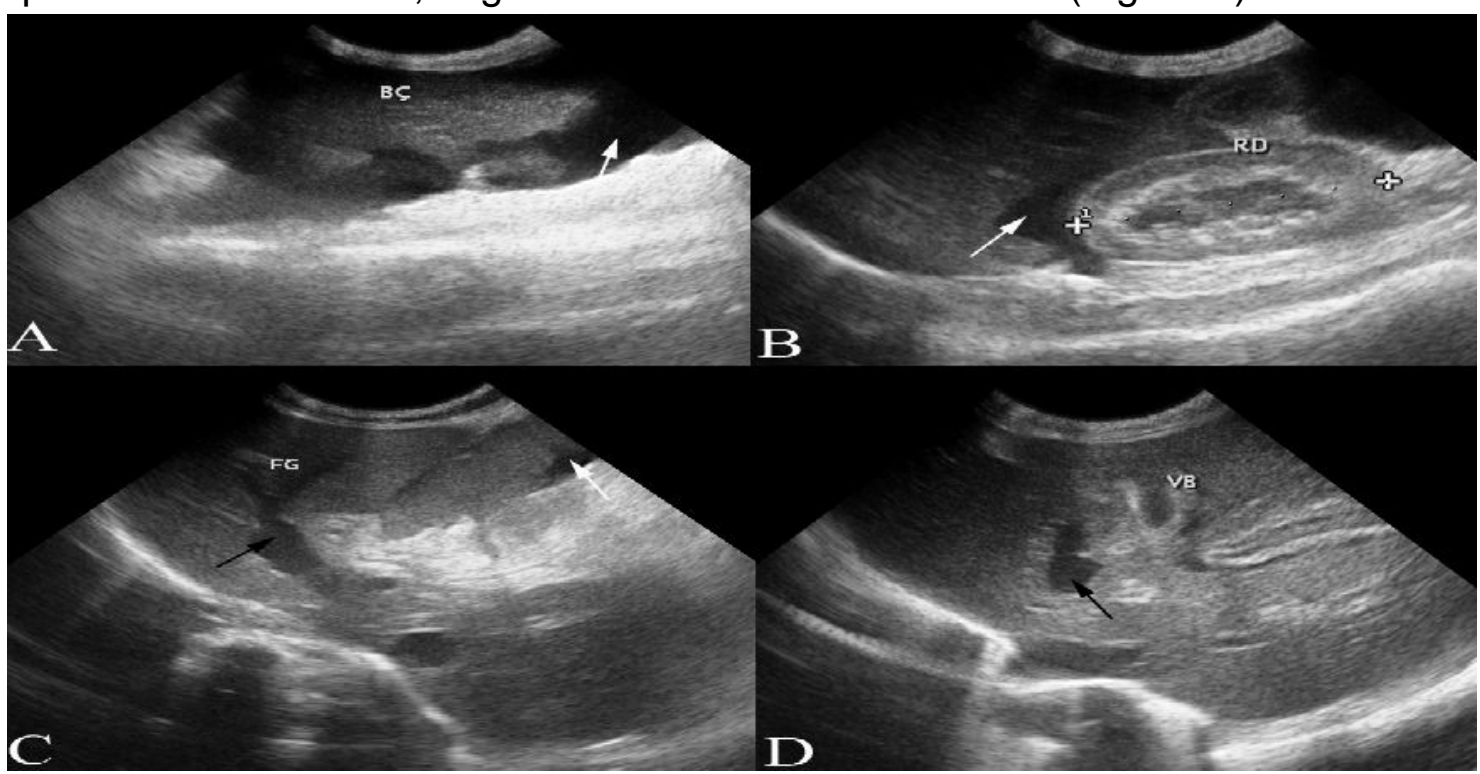

FIGURA 1. Exame ultrassonográfico em um cão da raça shih-tzu. (A) Baço com dimensões discretamente aumentadas, parênquima homogêneo e hiperecoico, sugestivo de congestão e inflamação e presença de liquido livre evidenciado pela seta. (B) Rim com arquitetura interna alterada, relação corticomedular irregular, cortical delgada, hipoecoica com ecotextura preservada, sinal da margem medular, divertículos irregulares e região medular heterogênea, sugestivo de nefrite congênita. Presença de liquido livre evidenciado pela seta. (C e D) Fígado aumentado, com bordos arredondados, parênquima homogêneo e bastante hiperecoico e vasos moderadamente dilatados (seta preta), sugestivo de congestão, colestase e hepatite. Presença de liquido livre (seta branca). Fonte: elaboração própria. 
No exame ecodopplercardiográfico, foi possível observar dilatação atrial e ventricular direita, com regurgitação de tricúspide, valva tricúspide de aspecto anormal, com folheto septal preso ao septo e folheto anterior maior que o normal e movimentação paradoxal do septo devido à sobrecarga de volume do ventrículo direito. O gradiente de pressão do fluxo de regurgitação da tricúspide $26 \mathrm{mmHg}$, caracterizando hipertensão pulmonar leve (Figura 2).

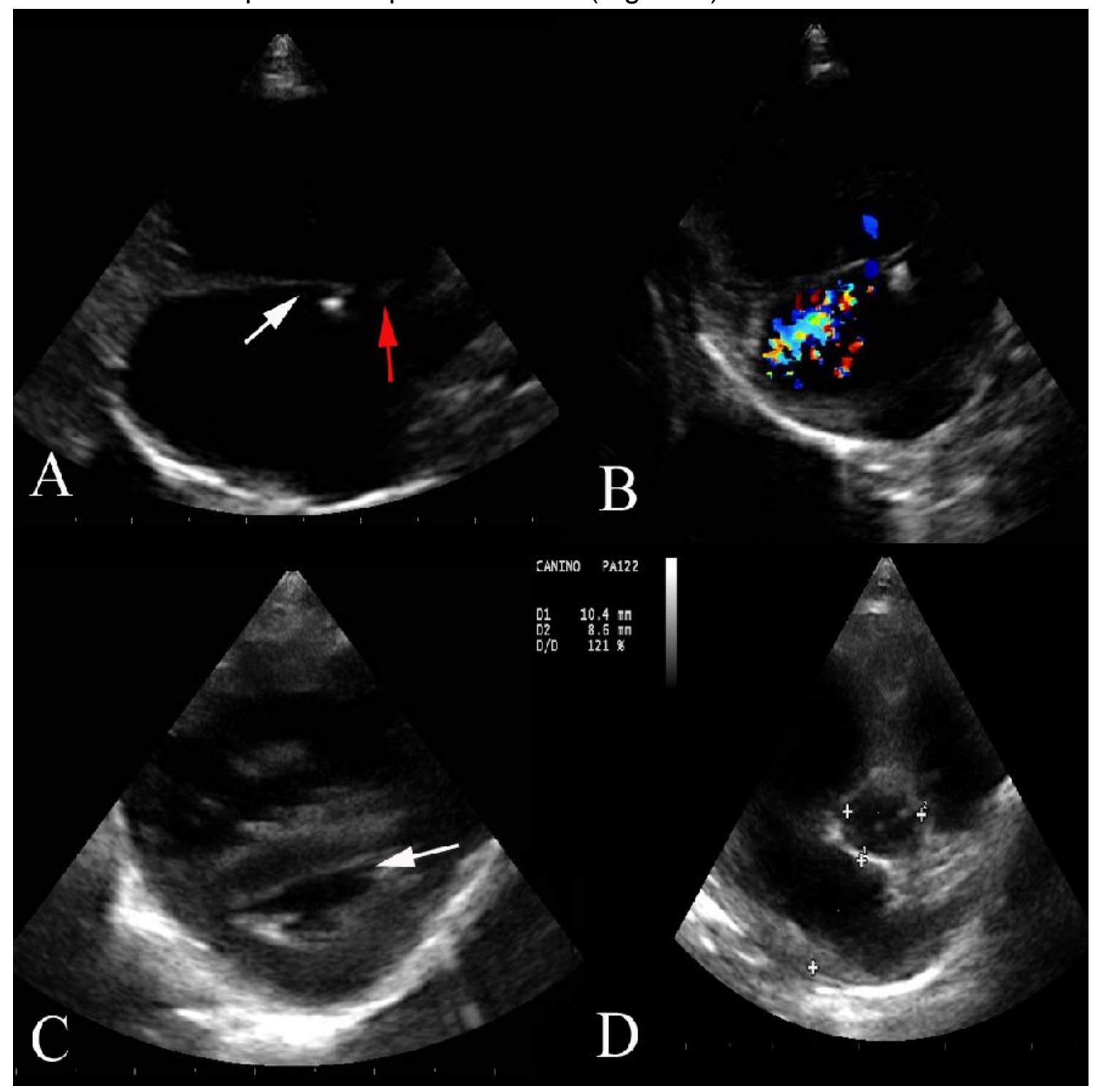

FIGURA 2. Exame ecodopplercardiográfico de um cão da raça shiht-zu de 2 meses, apresentando (A) Folheto anterior da valva tricúspide maior que o normal (seta branca) e folheto septal diminuído e preso ao septo (seta vermelha). (B) Regurgitação da valva tricúspide devido a não coaptação dos folhetos valvares, evidenciado pelo fluxo turbulento no Doppler colorido. (C) Movimentação paradoxal do septo secundário a sobrecarga de volume do ventrículo direito vista nas imagens transversais como um septo plano com um formato mais triangular da câmara ventricular esquerda (seta). (D) Relação do átrio esquerdo com a aorta normal $(1,21)$, demonstrando normalidade do lado esquerdo do coração.

\section{RESULTADOS E DISCUSSÃO}

A displasia de valva tricúspide é uma cardiopatia congênita pouco frequente em cães (NELSON; COUTO, 2009; ETTINGER; FELDMAN, 2010; OLIVEIRA et al., 2011; BEIJERINK et al., 2017). As cardiopatias congênitas 
apresentam uma frequência de $21,7 \%$ nos cães e a displasia de valva tricúspide apenas 3,1\%. (OLIVEIRA et al., 2011; BEIJERINK et al., 2017). De acordo com Schrope (2015), a afecção representa aproximadamente 2 a $7 \%$ de todas as malformações cardíacas congênitas em cães.

As raças mais acometidas são de grande porte, como Labrador, Pastor Alemão e Boxer, sendo os machos mais acometidos (OLIVEIRA et al., 2011). Além dessas raças, a patologia já foi relatada em cães da raça Dogue Alemão, Setter Irlandês, Old English Sheepdog e Weimaraner (BONAGURA, 1989; LIU; TILLEY, 1976; MOISE, 1989).

Até então, a afecção em cão da raça shih-tzu não havia sido relatada. No Brasil, a frequência desta patologia não é tão conhecida e sabe-se que pode haver variação na incidência e nas raças mais acometidas de acordo com cada região (SOUSA et al., 2006). A displasia de valva tricúspide já foi relatada em cães da raça Cocker Spaniel inglês, de cinco meses de idade, em cão sem raça definida de quatro meses (SOUSA et al., 2006), em Beagle de 11 meses (BRANCO et al., 2012), em um Boxer de cinco meses de idade (HUPPES, 2012) e em um Blue Heleer de 10 meses (MATTEUCCl et al., 2016).

Em um estudo realizado por Netuu et al. (2017), um cão macho de um ano de idade da raça Mastiff foi apresentado com anorexia, ascite, intolerância ao exercício e letargia. As radiografias revelaram derrame pleural moderado a grave, elevação traqueal, aumento do ângulo de bifurcação traqueal e edema pulmonar. A ultrassonografia abdominal revelou ascite e hepatomegalia grave. O ecocardiograma com Doppler revelou regurgitação da valva tricúspide com aumento atrial direito e deslocamento dos folhetos valvares para o ventrículo direito, indicando anomalia de ebstein. $O$ animal foi tratado com diuréticos, vasodilatadores e drogas inotrópicas por quatro semanas. O paciente morreu em casa após quatro semanas de tratamento.

A afecção foi relatada também em um cão da raça Bulldog inglês, de três meses de idade, macho, com dificuldade respiratória, intolerância ao exercício e aumento de volume abdominal. As radiografias revelaram radiopacidade pulmonar com padrão alveolar, cardiomegalia generalizada, elevação traqueal, deslocamento dorsal de traqueia. No exame ecocardiográfico, observou-se defeito septal ventricular, sobrecarga de volume, artéria pulmonar dilatada. O animal foi à óbito (AGUILAR et al., 2016).

De acordo com Beijerink et al. (2017), o aumento da prevalência da afecção em cães de raça sugere uma predisposição genética. No entanto, em um estudo realizado por Hokanson et al. (2019), a afecção foi diagnosticada em um cão de raça indefinida, macho, com 10 semanas de idade.

O primeiro relato de displasia de tricúspide em felinos foi descrito por Mantovani et al. (2017) em uma gata com quatro anos de idade apresentando apatia, perda de apetite e dispneia há cinco dias. Durante o exame físico observouse dispneia com presença de efusão pleural. O ecocardiograma revelou hipertrofia excêntrica do ventrículo direito e movimento septal paradoxal com remodelamento de câmaras cardíacas direitas. A valva tricúspide apresentou-se com perda de mobilidade de sua cúspide septal e espessamento de cordoalha tendínea da cúspide mural, com inserção anômala. Iniciou-se o tratamento com enalapril $(0,5 \mathrm{mg} / \mathrm{kg})$, furosemida $(0,5 \mathrm{mg} / \mathrm{kg})$ e pimobendan $(0,3 \mathrm{mg} / \mathrm{kg})$, pela via oral a cada 12 horas. A paciente apresentou remissão das manifestações clínicas, sobrevivendo por 50 dias após o diagnóstico. 
A afecção já foi relatada também em um bezerro da raça Red Angus, com dois meses de idade. $O$ animal apresentava taquicardia, taquipnéia, dispnéia e mucosas cianóticas. $\mathrm{Na}$ ausculta, presença de sopro sistólico apical direito grau 2/6. Radiografias torácicas revelaram cardiomegalia, afetando mais severamente o lado direito. O ecocardiograma demonstrou displasia da valva tricúspide, resultando em grave regurgitação tricúspide e dilatação do coração direito, além de forame oval patente. A necropsia confirmou a presença das anormalidades cardíacas mencionadas e revelou alterações pulmonares leves (MOREIRA et al., 2019).

Não foi encontrada relação da afecção com predisposição sexual. Em dois estudos, os homens pareciam ser mais afetados do que as mulheres (PASLAWSKA et al., 2013), enquanto em outro estudo (OLIVEIRA et al., 2011) mais fêmeas foram diagnosticadas com a afecção. Em um recente estudo de prevalência de cardiopatias congênitas em cães, ambos os sexos foram acometidos (SCHROPE, 2015).

De acordo com Lake-Bakaar et al. (2017), a displasia da valva tricúspide em cães engloba um espectro de malformações congênitas dos aparatos da valva tricúspide, incluindo espessamento focal ou difuso dos folhetos, subdesenvolvimento de cordas tendíneas, músculos papilares, evoluindo para agenesia de tecido valvar, resultando em insuficiência cardíaca ou raramente estenose.

Os sinais observados no exame clínico do animal do presente relato foram característicos da insuficiência cardíaca congestiva direita, corroborando com fontes na literatura (TILLEY; GOODWIN, 2002; MACDONALD, 2006). A alteração clínica mais comum é a presença de um sopro holossistólico na auscultação do foco tricúspide (FOX et al., 1999; NELSON; COUTO, 2009), também observada no animal do relato. De acordo com Beijerink et al. (2017), é comum a observação de anorexia e caquexia, além de distensão da veia jugular. Hepatomegalia e ascite podem ocorrer em caso de insuficiência cardíaca congestiva (BEIJERINK et al., 2017) corroborando com os dados observados no cão do presente relato.

Anemia, trombocitopenia, hipoalbuminemia e a presença de líquido abdominal do tipo transudato modificado podem ser observados nos achados laboratoriais em animais com displasia valvar tricúspide (SOUSA et al., 2006). Os achados hematológicos, exceto a trombocitopenia, e do líquido ascítico encontrados no presente relato corroboram com Sousa e colaboradores (2006) e Beijerink e colaboradores (2017).

O ecocardiograma é o exame indicado para o diagnóstico definitivo das cardiopatias congênitas (FOX et al., 1999; MACDONALD, 2006; NELSON; COUTO, 2009; ETTINGER; FELDMAN, 2010). As imagens ecocardiográficas que melhor possibilitam a avaliação da valva tricúspide são as obtidas na longitudinal quatro câmaras da paraesternal direita e apical quatro câmaras na paraesternal esquerda (MADRON et al., 2015).

$\mathrm{Na}$ displasia da válvula tricúspide, ocorrem alterações morfológicas nos folhetos da válvula atrioventricular direita, que causa uma menor coaptação dos folhetos valvares, levando a regurgitação de sangue para o átrio direito e sobrecarga de volume (MACDONALD, 2006; ETTINGER; FELDMAN, 2010; BEIJERINK et al., 2017). Há dilatação atrial e hipertrofia ventricular excêntrica, sinais clássicos da insuficiência cardíaca congestiva direita (FOX et al., 1999; NELSON; COUTO, 2009).

$\mathrm{Na}$ maioria dos casos, a valva displásica está espessada, porém móvel, e um ou mais folhetos estão fixados aos músculos papilares aumentados, diretamente ou por meio das cordas tendíneas encurtadas. Isso resulta no 
aparecimento do travamento ventral e na aposição incompleta dos folhetos durante a sístole ventricular (NYLAND; MATTOON, 2005; BEIJERINK et al., 2017). É comum visualizar dilatação atrioventricular direita pela sobrecarga de volume, bem como outras alterações já relatadas na doença mixomatosa de valva tricúspide (MADRON et al., 2015).

No estudo, foi observado aumento severo do átrio e do ventrículo direito. Nas imagens obtidas no corte transversal paresternal direito, foi possível observar que a sobrecarga do volume do ventrículo direito resultou em movimento paradoxal do septo e um ventrículo esquerdo comparativamente pequeno, o que corrobora com Friedman (1999). As alterações da valva tricúspide observadas neste caso foram similares a outras relatadas na literatura, que incluíam folhetos espessados, de tamanho anormal, com pouco movimento e coaptação (CAVE, 2001).

De acordo com Tierney et al. (2017), o prognóstico da afecção na medicina humana é de reservado a grave e o comprometimento hemodinâmico progressivo é comum. Na medicina veterinária, de acordo com Beijerink et al. (2017), a prevalência inicial nos cães é desconhecida. Em jovens e adultos, o prognóstico é semelhante ao dos humanos e depende principalmente na gravidade da malformação.

Cães com pequenas insuficiências geralmente têm uma vida normal. Um fluxo regurgitante de alta velocidade durante a sístole, insuficiência cardíaca direita e fibrilação atrial constituem um prognóstico ruim. Alguns cães podem ter uma boa qualidade da vida por vários anos sob medicação, enquanto outros podem ter uma rápida progressão da doença (BEIJERINK et al., 2017).

Em um estudo realizado por Navarro-Cubas et al. (2017), foi determinada a sobrevida e os fatores de risco de mortalidade por displasia da valva tricúspide em cães do Reino Unido. A arritmia mais comum foi a fibrilação atrial. O tempo médio de sobrevida do diagnóstico de displasia valvar de tricúspide foi de 2775 dias e do início do quadro de insuficiência cardíaca congestiva direita foi de 181 dias. A síncope foi o único fator de risco para falência cardíaca.

Neste estudo de cães do Reino Unido, a displasia da valva tricúspide era incomum, mas, quando agravava, frequentemente levava à insuficiência cardíaca congestiva. O prognóstico foi favorável para displasia tricúspide leve e moderada. O tempo de sobrevida foi reduzido com a insuficiência cardíaca congestiva do lado direito (NAVARRO-CUBAS et al., 2017).

O tratamento da afecção é de suporte. $O$ efeito do tratamento preventivo antes do início dos sinais clínicos é desconhecido (BEIJERINK et al., 2017). Os inibidores da enzima conversora da angiotensina (ECA) são aconselhados na tentativa de prevenir a progressão da insuficiência cardíaca congestiva. Fármacos inotrópicos positivos são recomendados para melhorar a função sistólica do ventrículo direito em casos de insuficiência cardíaca direita. Diuréticos como a furosemida são essenciais para controlar os sinais de congestão.

Uma dieta bem equilibrada com baixo teor de sódio é indicada. Em caso de apresentação aguda de dispneia e ascite, oferecer oxigenoterapia, toracocentese e/ou abdominocentese (PASLAWSKA et al., 2013; BEIJERINK et al., 2017). Em caso de presença de fibrilação atrial ou taquicardia supraventricular recomenda-se a utilização de digoxina, bloqueadores dos canais de cálcio e beta-bloqueadores (BEIJERINK et al., 2017).

O tratamento da regurgitação tricúspide devido à displasia de valva tricúspide na medicina humana envolve a valvoplastia cirúrgica ou a substituição valvar. Ambas biopróteses e próteses mecânicas são usadas para 
substituição artificial de válvulas. A principal vantagem da prótese mecânica em comparação com biopróteses é a durabilidade (BRISTOW et al., 2017).

Em um estudo realizado por Bristow et al. (2017), foi avaliado o resultado da substituição valvar em cães com displasia da valva tricúspide. O critério para escolha do paciente à substituição valvar foi regurgitação da valva tricúspide associada a sinais clínicos de comprometimento cardíaco. Nove cães de seis raças diferentes foram escolhidos, com média de um ano de idade. Oito válvulas pericárdicas bovinas e uma válvula aórtica suína foram utilizadas.

Uma complicação trans-operatória ocorreu, porém o animal sobreviveu. Complicações pós-operatórias ocorreram em seis cães, dos quais quatro foram fatais. Dos cinco cães, um apresentou morte por hemotórax dias depois. Os quatro cães restantes sobreviveram em média 533 dias e todos receberam a válvula de pericárdio bovino (BRISTOW et al., 2017).

Com base nos resultados do estudo de Bristow et al. (2017), a substituição valvar com prótese bovina ou suína em cães está associada a uma alta incidência de complicações. Conclui-se que a técnica utilizada é um tratamento de alto risco em cães.

Em um trabalho conduzido por Lake-Bakaar e colaboradores (2017), foi realizada valvoplastia em cinco cães da raça Labrador com estenose grave de valva tricúspide. Os cães apresentavam fraqueza, sincope periódica, distensão abdominal, letargia e intolerância ao exercício. O procedimento de valvoplastia com balão foi tentado em todos os cães e completado em quatro deles. A pressão atrial direita diminuiu em quatro animais. Todos, exceto um cão, tiveram melhora clínica após a valvoplastia, mas houve recidiva dos sinais clínicos em dois animais. A regurgitação tricúspide piorou em um cão, culminando em insuficiência cardíaca direita e eutanásia.

\section{CONCLUSÃO}

A displasia de tricúspide pode ocorrer em cão da raça shih-tzu, apesar de ser uma anormalidade cardíaca congênita pouco comum e com poucos casos relatados no Brasil. A presença de sinais clínicos compatíveis com doença cardíaca em cães filhotes e jovens levanta a possibilidade de doenças cardíacas congênitas, que devem ser investigadas mediante o exame clínico e exames complementares, sendo o ecocardiograma o mais indicado para um diagnóstico preciso.

\section{REFERÊNCIAS}

AGUILAR, G. F. RODRIGUEZ VELAZQUEZ, D. BARBOSA, M. Tricuspid Valve Dysplasia in English Bull Dog Breed: About a Case. EC Veterinary Science, v. 2, p. 237-241, 2016. Disponível em <https://core.ac.uk/download/pdf/84813095.pdf>

BECKER, A. E.; BECKER, M. J.; EDWARDS, J. E. Pathologic spectrum of dysplasia of the tricuspid valve. Features in common with Ebstein's malformation. Archives of pathology, v. 91, n. 2, p. 167-178, 1971. Disponível em: < https://europepmc.org/abstract/med/5099884>.

BEIJERINK N.J., OYAMA M.A., BONAGURA J.D. Congenital heart disease. In: Ettinger S.J., Feldman E.C., Côte E. (editors). Textbook of Veterinary Internal Medicine: Diseases of the Dog and Cat. Eighth edition, volume 2, Elsevier Saunders, Missouri, p. 1225-1227, 2017. 
BOON, J.A. Ecocardiografia bidimensional e em modo-M para o clínico de pequenos animais. 1a ed. São Paulo: Roca, 2005.

BOON, J. A.. Veterinary Echocardiography. lowa: Blackwell Publishing, 2011.

BRANCO, S. E. M. T., CAVALCANTI, G. A. O, CARNEIRO, R. A., BARACAT DE ARAÚJO, R. Displasia de tricúspide em cão da raça beagle - relato de caso. Archives of Veterinary Science, v. 17, n.1, p. 48-50, 2012. Disponível em: < https://revistas.ufpr.br/veterinary/issue/view/1347

http://dx.doi.org/10.5380/avs.v17i1e

BRISTOW, P., SARGENT, J., LUIS FUENTES, V., \& BROCKMAN, D. Outcome of bioprosthetic valve replacement in dogs with tricuspid valve dysplasia. Journal of Small Animal Practice, v. 58, n.4, p.205-210, 2017. Disponível em <https://core.ac.uk/download/pdf/79609444.pdf>. doi:10.1111/jsap.12630.

BONAGURA, J.D. Congenital heart diseases. In: ETTINGER, S.J. Textbook of veterinary internal medicine. 3.ed. Philadelphia, W.B. Saunders, 1989. 976 p.

CAVE, T. What was your diagnosis? Congenital tricuspid dysplasia with concurrent Ebstein's anomaly. The Journal of small animal practice, v. 42, n. 6, p. 311-314, 2001. Disponível em: <https://europepmc.org/abstract/med/11440395>.

ETTINGER, S.J.; FELDMAN, E.C. Tratado de medicina interna veterinária. 7a ed. Rio de Janeiro: Guanabara Koogan. 2010.

FILIPPI, L.H. O eletrocardiograma na medicina veterinária. 1a ed. São Paulo: Roca. 2011.

FRIEDMAN, W.F. Cardiopatia congênita na lactância e infância. In: BRAUNWALD, E. Tratado de medicina cardiovascular. São Paulo: Roca, Cap. 29. p.935-1027. 1999.

FOX, P.R, SISSON, D.D, MOISE, N.S. Textbook of canine and feline cardiology. 2a ed. Philadelphia: WB Saunders Company; 1999.

HOKANSON, C. M.; RHINEHART, J. D.; SCANSEN, B. A. Bidirectional flow across a perforate cor triatriatum dexter in a dog with concurrent pulmonary, tricuspid, and mitral valve dysplasia. Journal of Veterinary Cardiology, v. 21, p. 93-97, 2019. doi: https://doi.org/10.1016/j.jvc.2018.12.002

HUPPES, R.R. Insuficiência cardíaca congestiva secundária à anomalia de Ebstein em um boxer de cinco meses de idade-relato de caso. Revista Clínica Veterinária, v. 17, n. 97, p. 42-46, 2012. Disponível em http://www.revistaclinicaveterinaria.com.br/edicao/2012/marco-abril.html.

LAKE-BAKAAR, G.A., GRIFFITHS, L.G. AND KITTLESON, M.D. Balloon Valvuloplasty of Tricuspid Stenosis: A Retrospective Study of 5 Labrador Retriever Dogs. Journal of Veterinary Internal Medicine, v. 31, p. 311-315, 2017. Disponível em <https://onlinelibrary.wiley.com/doi/full/10.1111/jvim.14671> doi:https://doi.org/10.1111/jvim.14671 
LIU, S. K.; TILLEY, L. P. Dysplasia of the tricuspid valve in the dog and cat. Journal of the American Veterinary Medical Association, v. 169, n. 6, p. 623-630, 1976. Disponível em: <https://europepmc.org/abstract/med/134984>.

MACDONALD, Kristin A. Congenital heart diseases of puppies and kittens. Veterinary Clinics: Small Animal Practice, v. 36, n. 3, p. 503-531, 2006. Disponível em: <https://www.vetsmall.theclinics.com/article/S0195-5616(05)001920/abstract> doi: https://doi.org/10.1016/j.cvsm.2005.12.006

MADRON, E.; CHETBOUL, V.; BUSSADORI, C. Clinical Echocardiography of the Dog and Cat. Elsevier Health Sciences. 2015.

MALVINDI P.G., VIOLA N. Ebstein's anomaly: diagnosis and surgical treatment. Giornale Italiano di Cardiologia v. 16, p. 175-185, 2015. Disponível em <https://europepmc.org/abstract/med/25837461> doi:10.1714/1820.19828.

MANTOVANI, M. M., MARTINS GIMENES, A., RIBEIRO DE CASTRO, J., TEIXEIRA GOLDFEDER, G., MATIKO AKAO LARSSON, M. H., \& SARETTA SCHWARTZ, D. Tricuspid valve dysplasia in a domestic feline: case report. Semina: Ciências Agrárias, $\quad$ v. $38, \quad \mathrm{n} . \quad 2, \quad 2017 . \quad$ Disponível em: <https://www.redalyc.org/html/4457/445750711049/> DOI: 10.5433/16790359.2017v38n2p1087

MATTEUCCI, G., CHEROTO, A. L. L., YAMANISHI SHIMURA, K. M., \& GOMES, L. A. Estenose pulmonar e displasia de tricúspide: relato de caso em um cão da raça blue heeler. Veterinária e Zootecnia, v. 23, n. 2, p. 198-202, 2016. Disponível em: < https://www.cabdirect.org/cabdirect/abstract/20163216811>.

MEURS, K. M. Genetics of cardiac disease in the small animal patient. Veterinary Clinics of North America: Small Animal Practice, v. 40, n. 4, p. 701-715, 2010. Disponível em: <https://www.vetsmall.theclinics.com/article/S0195-5616(10)000343/pdf> doi: https://doi.org/10.1016/j.cvsm.2010.03.006.

MOISE, N.S. Doppler echocardiographic evaluation of congenital cardiac disease. Journal of Veterinary Internal Medicine, v.3, n.4, p .195-207, 1989. doi: https://doi.org/10.1111/j.1939-1676.1989.tb00858.x

MOREIRA, A. D., GRINT, K., STEPIEN, R., SHAW, G., \& PEEK, S. Tricuspid valve dysplasia and a patent foramen ovale resulting in severe tricuspid regurgitation and right-heart dilation in a Red Angus calf. Journal of Veterinary Cardiology, v. 21,p. 28-33. 2019. doi: https://doi.org/10.1016/j.jvc.2018.10.005

NAVARRO-CUBAS, X., PALERMO, V., FRENCH, A., SANCHIS-MORA, S., \& CULSHAW, G. Tricuspid valve dysplasia: A retrospective study of clinical features and outcome in dogs in the UK. Open veterinary journal, v.7, n.4, p. 349-359, 2017. DOI: http://dx.doi.org/10.4314/ovj.v7i4.11

NELSON, R.W.; COUTO, C.G. ; Medicina interna de pequenos animais. 4a ed. São Paulo: Elsevier, 2009.

NETUU, S., KUMAR, U. S., ROHIT, T., SINGH, R. S., \& ARUN, A. A Rare Case of Ebstein Anomaly in a French Mastiff Dog. Cardiovasc Ther, v. 7, n.2, p. 555706, 2017. DOI: 10.19080/JOCCT.2017.07.555706 
NYLAND, T. G.; MATTOON, J. S. Ultrassom diagnóstico em pequenos animais. Roca, São Paulo, p. 161-198, 2005.

OLIVEIRA, P., DOMENECH, O., SILVA, J., VANNINI, S., BUSSADORI, R., et al. Retrospective Review of Congenital Heart Disease in 976 Dogs. Journal of Veterinary Internal Medicine, v.25, p.477-483. Disponível em <https://onlinelibrary.wiley.com/doi/epdf/10.1111/j.1939-1676.2011.0711.x> doi: https://doi.org/10.1111/j.1939-1676.2011.0711.x

OLIVIER, N. B. Congenital heart disease in dogs. In Fox PR (ed): Canine and Feline Cardiology. New York: Churchill Livingstone, p 357-389, 1988. Disponível em: <https://onlinelibrary.wiley.com/doi/pdf/10.1111/j.1748-5827.1989.tb01485.x> doi: https://doi.org/10.1111/j.1748-5827.1989.tb01485.x

PASLAWSKA U., NOSZCZUK-NOWAK A., JANISZEWSKI A., NICPON J. Tricuspid dysplasia in dogs. Bulletin of the Veterinary Institute in Pulawy v.57, p. 123-126, 2013. Disponível em <https://www.degruyter.com/downloadpdf/j/bvip.2013.57.issue1/bvip-2013-0023/bvip-2013-0023.pdf> DOI: 10.2478/bvip-2013-0023.

SOUSA, M.G., GERARDI, D.G., ALVES, R.O, CAMACHO, A. A. Tricuspid valve dysplasia and Ebstein's anomaly in dogs: case report. Arquivo Brasileiro de Medicina Veteterinária e Zootecnia., v.58, n.5, p.762-767, 2006. Disponível em <http://www.scielo.br/pdf/abmvz/v58n5/10.pdf> doi: http://dx.doi.org/10.1590/S010209352006000500010

SCHROPE, D.P. Prevalence of congenital heart disease in 76,301 mixed-breed dogs and 57,025 mixed-breed cats. Journal of Veterinary Cardiology, v. 17, p. 192-202, 2015. doi:https://doi.org/10.1016/j.jvc.2015.06.001

THRALL, D.E. Diagnóstico de radiologia veterinária. 5a ed. São Paulo: Elsevier, 2010.

TIERNEY, E.S.S., MCELHINNEY, D.B., FREUD, L.R., TWORETZKY, W., CUNEO, B.F., ESCOBAR-DIAZ, M.C., PUCHALSKI, M.D. Assessment of progressive pathophysiology after early prenatal diagnosis of the Ebstein anomaly or tricuspid valve dysplasia. The American journal of cardiology, v. 119, n. 1, p. 106-111, 2017. doi: https://doi.org/10.1016/j.amjcard.2016.09.022.

TILLEY, L.P.; GOODWIN, J. In: STRICKLAND, K. N. Livro Manual de Cardiologia para Cães e Gatos. Capitulo 17, P. 323-345, 2002.

WANG, S., FREUD, L.R., DETTERICH, J. A., MOON-GRADY, A.J., DONOFRIO, M. T., JAEGGI, E.T., VAN DER VELDE, M.E. Extracardiac Doppler Abnormalities Are Associated With Perinatal Mortality in Fetuses With Ebstein Anomaly and Tricuspid Valve Dysplasia. Circulation, v. 138, n. 1, p. A15904-A15904, 2018. 